Dr. Miao Mi

China Media Centre, CAMRI, University of Westminster

Ace.maggie@gmail.com

$+44(0) 7775551383$

Professor Hugo de Burgh

Professor of Journalism, Director,

China Media Centre, CAMRI, University of Westminster

H.De-Burgh@westminster.ac.uk

\title{
How Newspapers in China and the UK Respond to the Development of Communication Technologies: a Comparative Study
}

\begin{abstract}
This study investigates whether Internet technology and New Media innovations have been homogenizing British and Chinese press journalism, and, if not, in which ways and why they have responded differently.

The basic assumption of the thesis has been raised from Fidler's mediamorphosis theory and it argues that the transformation of mass media is normally brought about by a complex interplay between many variables (1996). To do this, the study examines the complex processes through which news organizations have been repositioning their brand identity and value, and through which journalistic professionals have been redefining the notion of their craft and their work practices in the UK and China. It first takes a historical perspective identifying the commonalities and differences between press journalism of the two countries as well as social and economic conditions that have influenced the ways in which newspapers have responded to the development of communication technologies. The thesis then selects four well-established newspapers from the UK and China and compares the commonalities and differences between the online content they produced, their editorial organizational patterns, established journalistic culture in the four selected newsrooms. This study in the end discussed the impacts of the complex interplay between historical journalistic traditions, commercial drives and organizational structure as well as journalistic practices upon the ways in which newspaper organizations in the two countries have responded to Internet technology and New Media innovations.
\end{abstract}

This study finds that although all newspapers claim to be multi-platform content providers, the two British newspapers, The Guardian and the Daily Mail, have enhanced their brand value on the Internet by extending their journalistic values and good practice online, while the two Chinese newspapers, the China Youth Daily and the Southern City Daily, have faced a decline in brand credibility, a decrease in journalistic integrity and an erosion of selfsatisfaction among Party journalists. This study identifies the changes in organizational structure, as well as the negotiations between the Internet's social and technical dimensions with newsroom culture; and it goes on to evaluate how newspapers have managed to achieve a good online strategy and excellent online journalistic practice, and have enhanced journalists' self-perception. 
However, the findings have also shown that there is a danger of deterioration in the quality of journalism, and the reasons have been identified as: 1 . Requirements for multimedia skills; 2 . increased competition between 24-hour news channels, online news portals and social media platforms; 3 . the replacement of traditional editorial judgments and journalistic practice through the availability of various technical supplements.

The study makes two contributions to knowledge. First, the comparison between the UK and China is a starting-point in seeking a new approach to the understanding of media convergence and New Media phenomena, and a new way of reasoning as to how the interplay between technological, cultural, commercial and political pressures might shape journalism in terms of newsroom practice/routines and organizational structure in different countries. Second, it is the first doctoral study in the UK and other English-speaking countries to compare how newspapers organization practice online journalism in the UK and China as responses to the development of Internet technologies and New Media innovations, not only by providing empirical evidence, but also by providing quantitative content analysis of newspapers' online websites and a comparison between each newspaper's online and offline versions. It will bring the understanding of Chinese journalism up-to-date.

Keywords: Comparative Journalism Studies, Press Journalism in China and the UK, Newsroom Convergence, News Gathering and Production 